\title{
The Initial Boundary Value Problem for the Equations of Ideal Magneto-Hydrodynamics
}

\author{
Taku Yanagisawa \\ (Received October 15, 1986, Revised June 25, 1987)
}

\section{$\S 1$. Introduction and Results.}

We consider the initial boundary value problem for the equations of ideal magneto-hydrodynamics :

$$
\begin{aligned}
& \begin{cases}\text { (a) } & \rho_{p}\left(\partial_{t}+(u \cdot \nabla)\right) p+\rho \operatorname{div} u=0 \\
\text { (b) } & \rho\left(\partial_{t}+(u \cdot \nabla)\right) u+\nabla p+\mu H \times \operatorname{curl} H=0 \\
\text { (c) } \partial_{t} H-\operatorname{curl}(u \times H)=0\end{cases} \\
& \left.(p, u, H)\right|_{t=0}=\left(p_{0}, u_{0}, H_{0}\right) \\
& u \cdot n=0, H \times n=g \\
& \text { in }[0, T] \times \Omega \text {, } \\
& \text { in } \Omega \text {, } \\
& \text { on }[0, T] \times \Gamma \text {. }
\end{aligned}
$$

Here $\Omega$ is a bounded domain in $R^{3}$ with $C^{\infty}$ boundary $\Gamma, T$ a given positive constant and $n=n(x)={ }^{t}\left(n_{1}, n_{2}, n_{3}\right)$ denotes the unit outward normal at $x \in$ $\Gamma$. Pressure $p=p(t, x)$, velocity $u=u(t, x)={ }^{t}\left(u_{1}, u_{2}, u_{3}\right)$ and the magnetic field $H=H(t, x)={ }^{t}\left(H_{1}, H_{2}, H_{3}\right)$ are unknowns. The permeability $\mu$ is supposed to be constant. We also suppose that density $\rho>0$ is a smooth known function of $p>0$ i.e. $\rho=\rho(p)$; and $\rho_{p}=\partial \rho / \partial p . g=g(t, x)={ }^{t}\left(g_{1}, g_{2}, g_{3}\right)$ is a given function on $[0, T] \times \Gamma . \partial_{t}=\partial / \partial t, \partial_{i}=\partial / \partial x_{i}(i=1,2,3), \nabla=\left(\partial_{1}, \partial_{2}, \partial_{3}\right)$, $(u \cdot \nabla)=\sum_{i=1}^{3} u_{i} \cdot \partial_{i}$ and $\cdot, \times$ denote scalar and vector product, respectively.

We assume that the initial data $p_{0}$ and $H_{0}$ satisfy

(1.5) $\operatorname{div} H_{0}=0$ in $\Omega$,

(1.6) $\quad \inf _{x \in \Gamma}\left|H_{0} \cdot n\right| \geqq c_{2}>0$.

Here $c_{1}$ and $c_{2}$ are positive constants. The assumptions (1.4), (1.5) guarantee the equations (1.1) to be a quasilinear symmetric hyperbolic system.

Our purpose of this paper is to show a local in time existence theorem for the initial boundary value problem (1.1)-(1.3).

THEOREM. Let $m$ be an integer $\geqq 3$. Assume that $g \in \dot{Y}_{m}(T)$ and the 
initial data $\left(p_{0}, u_{0}, H_{0}\right) \in H^{m}(\Omega)$ satisfies the assumptions (1.4), (1.5), (1.6) and the compatibility conditions of order $m$.

Then there exists a positive constant $T_{0}$ depending only on $\left\|\left(p_{0}, u_{0}, H_{0}\right)\right\|_{m}$, $\|g\|_{Y_{m}(T)}, c_{1}, c_{2}, m, \Omega$ such that the initial boundary value problem $(1,1),(1.2)$ and (1.3) has a unique solution which belongs to $X_{m}\left(T_{0}\right)$.

As to the definitions of the compatibility conditions and the function spaces $X_{m}(T)$ and $\dot{Y}_{m}(T)$, see (2.3), (2.4), (2.5) and (2.6) in $\S 2$.

We remark that this result can be extended to the nonisentropic case without any essential modification of the proof.

The initial boundary value problem for the compressible Euler equations under the solid-wall boundary condition $u \cdot n=0$ (i.e. (1.1)-(1.3) with $H=$ 0 ) is a typical characteristic initial boundary value problem for quasilinear symmetric hyperbolic systems and has been studied by many authors. (For example, c.f. [1], [3], [10] and [11].)

If one takes account of the effect of the magnetic field, one must consider the equations (1.1). However there seems to be no literature on the existence problem for (1.1). Here, we make some remarks on the boundary conditions. As the boundary conditions for $H$, we take $H \times n=g$ so that the solution is unique (see, Lemma 5.3). The assumption (1.6), imposed on $H_{0}$, is needed to guarantee the rank of the boundary matrix to be 6 on the boundary (see, (3.8)). It should be remarked that the assumptions (1.5) and (1.6) imply that $\Omega^{c}$ consists of at least two components.

The proof of Theorem proceeds via iteration scheme which involves the following steps. At first, following [10], we modify the equations (1.1) to make the boundary noncharacteristic (see, $(3.9)_{\varepsilon}$ ). We next establish the uniform estimates of the solution of the modified equations under the same initial boundary conditions (1.2) and (1.3). These uniform estimates are achieved by making use of a special structure of the modified equations and the fact that the rank of the boundary matrix is 6 on the boundary. Finally by taking a limit of these solutions, we get the solution of the original initial boundary value problem (1.1)-(1.3).

\section{$\S 2$. Preliminaries.}

In this section, we define some function spaces and the compatibility conditions and present the basic inequalities used in this paper.

$H^{m}(\Omega)$ denotes a usual scalar or vector valued Sovolev space of order $m$ equipped with inner product $(,)_{m}$ and norm $\|\cdot\|_{m}$

$$
(f, g)_{m}=\sum_{|\alpha| \leqq m} \int_{\Omega} \partial^{\alpha} f \cdot \partial^{\alpha} g \mathrm{~d} x,
$$




$$
\|f\|_{m}^{2}=\sum_{|\alpha| \leqq m} \int_{\Omega}\left|\partial^{\alpha} f\right|^{2} \mathrm{~d} x,
$$

where $\partial^{\alpha}=\partial_{1}^{\alpha_{1}} \partial_{2}^{\alpha_{2}} \partial_{3}^{\alpha_{3}}$ for $\alpha=\left(\alpha_{1}, \alpha_{2}, \alpha_{3}\right),|\alpha|=\alpha_{1}+\alpha_{2}+\alpha_{3}$.

We also use Sobolev space of order $s$ ( $s$ : real number) on the boundary $H^{s}(\Gamma)$ with norm $\|\cdot\|_{H}{ }^{s(\Gamma)}$.

The following well-known inequalities in three dimension are basic in this paper (c. f. [4] and Appendix B of [10]):

$$
\|f\|_{c^{j}(\bar{\Omega})} \leqq c\|f\|_{j+2} \text { for } f \in H^{j+2}(\Omega) \text { with } j \geqq 0,
$$

and

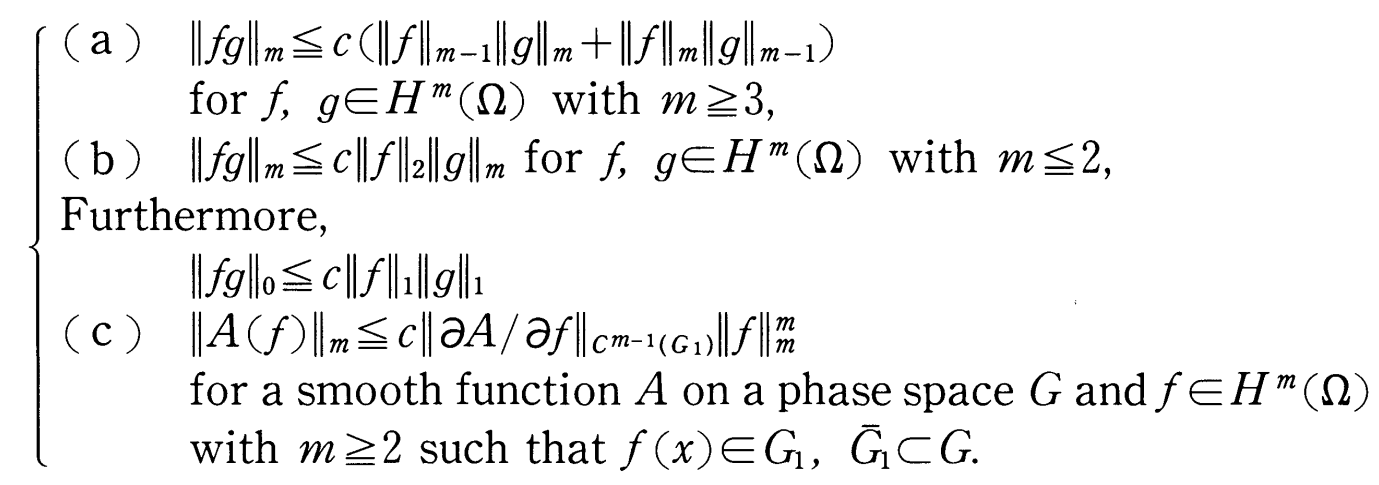

Here $\|\cdot\|_{C^{j}(K)}$ denotes $C^{j}$ norm on the set $K$ and $c$ is a constant depending only on $m$ and $\Omega$.

For a Banach space $B$, let $C^{j}(0, T ; B)$ be the set consisting of $j$-times continuously differentiable functions of $t \in[0, T]$ with values in $B$. Then we define

$$
\begin{aligned}
& X_{m}(T)=\bigcap_{j=0}^{m} C^{j}\left(0, T ; H^{m-j}(\Omega)\right), \\
& Y_{m}(T)=\bigcap_{j=0}^{m} C^{j}\left(0, T ; H^{m-j+1 / 2}(\Gamma)\right) \cap C^{m+1}\left(0, T ; H^{1 / 2}(\Gamma)\right),
\end{aligned}
$$

with norms

$$
\begin{aligned}
& \|u\|_{X_{m}(T)}=\sup _{t \in[0, T]}\|u(t)\|_{m}=\sup _{t \in[0, T]}\left(\sum_{j=0}^{m}\left\|\partial_{t}^{j} u(t)\right\|_{m-j}\right), \\
& \|g\|_{Y_{m}(T)}=\sup _{t \in[0, T]}\left(\sum_{j=0}^{m}\left\|\partial_{t}^{j} g(t)\right\|_{H^{m-j+1 / 2(\Gamma)}}+\left\|\partial_{t}^{m+1} g(t)\right\|_{H^{1 / 2}(\Gamma)}\right),
\end{aligned}
$$

where $u(t)$ stands for $u(t, x)$ for fixed $t$. Moreover we define

$$
\dot{Y}_{m}(T)=\left\{g \in Y_{m}(T) ; g \cdot n=0 \text { on }[0, T] \times \Gamma\right\} .
$$

Finally we say that the initial data $\left(p_{0}, u_{0}, H_{0}\right)$ satisfy the compatibility conditions of order $m$ for the equations (1.1) and the boundary conditions (1. 3), if 


$$
\begin{aligned}
& \partial_{t}^{k} u(0) \cdot n=0, \partial_{t}^{k} \mathrm{H}(0) \times n=\partial_{t}^{k} g(0) \text { on } \Gamma, \\
& \text { for } k=0,1, \ldots, m-1 .
\end{aligned}
$$

Here the terms $\partial_{t}^{k} \mathrm{u}(0), \partial_{t}^{k} \mathrm{H}(0)$. are calculated from (1.1) and (1.2), and are then expessed by initial data and their derivatives. For instance,

$$
\begin{aligned}
& \partial_{t} u(0)=-\left(u_{0} \bullet \nabla\right) u_{0}-\rho\left(p_{0}\right)^{-1}\left(\nabla p_{0}+\mu H_{0} \times \operatorname{curl} H_{0}\right), \\
& \partial_{t} H_{0}=\operatorname{curl}\left(u_{0} \times H_{0}\right) .
\end{aligned}
$$

\section{$\S 3$. A modification of the equations (1.1) to the noncharacteristic case.}

At first, we remark that (1.1)(c) is equivalent to

(1. 1)(c $)^{\prime}\left(\partial_{t}+(u \cdot \nabla)\right) H-(H \cdot \nabla) u+H \operatorname{div} u=0$ in $[0, T] \times \Omega$,

under the first condition of (1.3) and (1.5); This can be seen from wellknown formulae from vector analysis

$-\operatorname{curl}(u \times H)=(u \cdot \nabla) H-(H \cdot \nabla) u+H \operatorname{div} u-u \operatorname{div} H$, $\operatorname{div} \operatorname{curl}=0$.

Thus we consider the system of the equations $(1.1)(a)(b)(c)^{\prime}$ which is symmetric.

In order to make the boundary conditions homogeneous we construct an extension $G$ of $g$ as follows.

Lemma 3.1. For a function $g \in \dot{Y}_{m}(T)$, there exists an extension $G \in$ $\bigcap_{j=0}^{m} C^{j}\left(0, T ; H^{m-j+1}(\Omega)\right) \cap C^{m+1}\left(0, T ; H^{1}(\Omega)\right)$ such that

(a) $\operatorname{div} G=0$ in $[0, T] \times \Omega$,

(b) $G \cdot n=0, \mathrm{G} \times \mathrm{n}=g$ on $[0, T] \times \Gamma$,

(c) $\sup _{t \in[0, T]}\left(\sum_{j=0}^{m}\left\|\partial_{t}^{j} G(t)\right\|_{m-j+1}+\left\|\partial_{t}^{m+1} G(t)\right\|_{1}\right) \leqq c\|g\|_{Y_{m}(T)}$.

PROOF. Since $(n \times g) \cdot n=0$, it follows from the results of $\mathrm{L}$.

Cattabriga [2] that there exists an extension $G \in \bigcap_{j=0}^{m} C^{j}\left(0, T ; H^{m-j+1}(\Omega)\right)$ $\cap C^{m+1}\left(0, T ; H^{1}(\Omega)\right)$ satisfying $G=n \times g$ on $[0, T] \times \Gamma$, (a) and (c). Furthermore since $g \cdot n=0$ we can see that $(n \times g) \times n=g$, which completes the proof of $(b)$.

Set $\tilde{H}=H-G$. Then $(p, u, \tilde{H})$ is a solution of the initial boundary value problem: 


$$
\begin{aligned}
& \text { (a) } \rho_{p}\left(\partial_{t}+(u \cdot \nabla)\right) p+\rho \operatorname{div} u=0 \\
& \text { (b) } \rho\left(\partial_{t}+(u \cdot \nabla)\right) u+\nabla p+(\tilde{H}+G) \times \operatorname{curl} \tilde{H}+\tilde{H} \times \operatorname{curl} G \\
& =-G \times \operatorname{curl} G \\
& \text { (c })^{\prime}\left(\partial_{t}+(u \cdot \nabla)\right) \tilde{H}-((\tilde{H}+G) \cdot \nabla) u+(\tilde{H}+G) \operatorname{div} u+(u \cdot \nabla) G \\
& =-\partial_{t} G \\
& \text { in }[0, T] \times \Omega \text {, } \\
& \text { (3.2) }\left.(p, u, \tilde{H})\right|_{t=0}=\left(p_{0}, u_{0}, \tilde{H}_{0}\right), \tilde{H}_{0}=H_{0}-G(0) \text { in } \Omega \text {, } \\
& \text { (3.3) } u \cdot n=0, \tilde{H} \times n=0 \text { on }[0, T] \times \Gamma \text {. }
\end{aligned}
$$

Here we assumed, without loss of generality, that $\mu=1$; take a normalization if necessary.

By (1.5), (1.6), (2.6) and Lemma 3.1, we know that the initial data $U_{0}=\left(p_{0}, u_{0}, \tilde{H}_{0}\right)$ satisfy $(1.4)$ and

(3.4) $\operatorname{div} \tilde{H}_{0}=0$ in $\Omega$,

$$
\inf _{x \in \Gamma}\left|\tilde{H}_{0} \cdot n\right| \geqq c_{2} \text {, }
$$

$$
\partial_{t}^{k} u(0) \cdot n=0, \partial_{t}^{\mathrm{k}} \tilde{H}(0) \times n=0 \text { on } \Gamma, \text { for } k=0,1, \ldots, m-1 .
$$

For simplicity we will write again $H$ instead of $\tilde{H}$. To clarify the structure of this initial boundary value problem it is convenient to express the system (3.1) in the matrix form:

$$
A_{0}(U) \partial_{t} U+\sum_{j=1}^{3} A_{j}(U) \partial_{j} U+B(t, x) U=F(t, x) .
$$

Here $U={ }^{t}(p, u, H)$ and $A_{0}(U), A_{j}(U)(j=1,2,3),. F(t, x)$ are given explicitly by

$$
\begin{aligned}
& A_{0}(U)=\left[\begin{array}{ccc}
\rho^{-1} \rho_{p} & & 0 \\
& \rho I_{3} & \\
0 & & I_{3}
\end{array}\right], A_{j}(U)=\left[\begin{array}{ccc}
\rho^{-1} \rho_{p} u_{j} & { }^{t} e_{j} & 0 \\
e_{j} & \rho u_{j} I_{3} & D_{j} \\
0 & D_{j} & u_{j} I_{3}
\end{array}\right], \\
& D_{1}=\left[\begin{array}{ccc}
0 & 0 & 0 \\
H_{2}+G_{2} & -H_{1}-G_{1} & 0 \\
H_{3}+G_{3} & 0 & -H_{1}-G_{1}
\end{array}\right], D_{2}=\left[\begin{array}{ccc}
-H_{2}-G_{2} & H_{1}+G_{1} & 0 \\
0 & 0 & 0 \\
0 & H_{3}+G_{3} & -H_{2}-G_{2}
\end{array}\right] \text {, } \\
& D_{3}=\left[\begin{array}{ccc}
-H_{3}-G_{3} & 0 & H_{1}+G_{1} \\
0 & -H_{3}-G_{3} & H_{2}+G_{2} \\
0 & 0 & 0
\end{array}\right], F(t, x)=\left[\begin{array}{c}
0 \\
-G \times \operatorname{curl} G \\
-\partial_{t} G
\end{array}\right] \text {, }
\end{aligned}
$$

where $I_{k}$ is the $k \times k$ unit matrix, $e_{1}={ }^{t}(1,0,0)$ e. t. c., and $B(t, x)$ is the matrix which consists of first order derivatives of $G$.

By definition, the boundary matrix $A_{n}(U)$ is given by 


$$
\begin{aligned}
A_{n}(U) & =\sum_{j=0}^{3} n_{j} \cdot A_{j}(U) \\
& =\left[\begin{array}{ccc}
\rho^{-1} \rho_{p} u \cdot n & { }^{t} n & 0 \\
n & \rho u \cdot n I_{3} & { }^{t} \mathrm{D}_{n} \\
0 & D_{n} & \mathrm{u} \cdot \mathrm{nI}_{3}
\end{array}\right] \text { on } \Gamma,
\end{aligned}
$$

where $D_{n}=\left(-(H+G) \cdot n \delta_{i j}+n_{i}\left(H_{j}+G_{j}\right) \mid i \rightarrow 1,2,3, j \downarrow 1,2,3\right)$. Because of the boundary condition $u \cdot n=0, A_{n}(U)$ is singular on $\Gamma$, namely the boundary is characteristic for (3.1). Thus we consider the modified equations of (3.1) with a positive parameter $\varepsilon$ under the same initial boundary conditions (3.2) and (3.3) (cf. [10]):

$$
\begin{gathered}
\left\{\begin{array}{cc}
\text { (a) } \quad \partial \rho_{\varepsilon} / \partial p\left(\partial_{t}+\left(u_{\varepsilon} \cdot \nabla\right)\right) p_{\varepsilon}+\rho_{\varepsilon} \operatorname{div} u_{\varepsilon}=0, \\
\text { (b) } \rho_{\varepsilon}\left(\partial_{t}+\left(u_{\varepsilon} \cdot \nabla\right)\right) u_{\varepsilon}+\nabla p_{\varepsilon}+\left(H_{\varepsilon}+G\right) \times \operatorname{curl} H_{\varepsilon} \\
\quad+H_{\varepsilon} \times \operatorname{curl} G=-G \times \operatorname{curl} G, \\
\text { (c) } \begin{array}{r}
\left(\partial_{t}+\left(u_{\varepsilon} \cdot \nabla\right)\right) H_{\varepsilon}-\left(\left(H_{\varepsilon}+G\right) \cdot \nabla\right) u+\left(H_{\varepsilon}+G\right) \operatorname{div} u_{\varepsilon} \\
\quad+\left(u_{\varepsilon} \cdot \nabla\right) G+\varepsilon(\boldsymbol{n} \cdot \nabla) H_{\varepsilon}=-\partial_{t} G+\varepsilon(\boldsymbol{n} \cdot \nabla) \hat{H},
\end{array}
\end{array}\right. \\
\rho_{\varepsilon}=\rho\left(p_{\varepsilon}\right) . \quad
\end{gathered}
$$

Here $\boldsymbol{n}$ is an extension in $C^{\infty}(\bar{\Omega})$ of normal vector $n$ to $\bar{\Omega}$; and $\hat{H} \in$ $H^{m}([0, T] \times \Omega)$ is an extension of $\partial_{t}^{k} H(0) \in H^{m-k}(\Omega), k=0,1, \ldots, m-1$, to $[0, T] \times \Omega$ such that

$$
\left\{\begin{array}{l}
\partial_{t}^{k} \hat{H}(0)=\partial_{t}^{k} H(0) \text { on } \Omega \text { for } k=0,1, \ldots, m-1, \\
\|\hat{H}\|_{H^{m}([0, T] \times \Omega)} \leqq c\left\|U_{0}\right\|_{m},
\end{array}\right.
$$

where $\partial_{t}^{k} H(0)$ is recursively defined through the equations (3.1) and the data (3.2). (For the construction of this extension, see Theorem 2. 5.7 of [5].)

REMARK 3.1. We note that the compatbility conditions (3.6) is still satisfied for $(3.9)_{\varepsilon}$.

Now the boundary matrix $A_{n}^{\varepsilon}(U)$ for the modified equations $(3.9)_{\varepsilon}$ is expressed by

$$
A_{n}^{\varepsilon}(U)=\left[\begin{array}{ccc}
\rho^{-1} \rho_{p} u \cdot n & { }^{t} n & 0 \\
n & \rho u \cdot n I_{3} & { }^{t} D_{n} \\
0 & D_{n} & (u \cdot n+\varepsilon) I_{3}
\end{array}\right] .
$$

We shall show that the $A_{n}^{\varepsilon}(U)$ has the following properties.

Lemma 3.2. Let $U^{\prime}(t, x)={ }^{t}\left(p^{\prime}(t, x), u^{\prime}(t, x), H^{\prime}(t, x)\right)$ satisfy that $\left.u^{\prime} \cdot n\right|_{\Gamma}=0, H^{\prime} \times\left. n\right|_{\Gamma}=0$ and $\inf _{x \in \Gamma}\left|H^{\prime} \cdot n\right| \geqq \tilde{c}>0$ on $[0, T]$. 
The Initial Boundary Value Problem for the Equations

Then the following holds:

(1) For $\varepsilon>0, A_{n}^{\varepsilon}\left(U^{\prime}\right)$ is nonsingular on $\Gamma$.

(2) For $\varepsilon \geqq 0$ and fixed $(t, x) \in[0, T] \times \Gamma$, the linear subspace, $N=\left\{U={ }^{t}(p, u, H) \mid u \cdot n(x)=0, H \times n(x)=0.\right\}$ of $R^{7}$ is maximally nonnegative with respect to $A_{n}^{\varepsilon}\left(U^{\prime}(t, x)\right)$.

Proof. Let $U={ }^{t}(p, u, H)$ satisfy $A_{n}^{\varepsilon}\left(U^{\prime}\right) U=0$ on $[0, T] \times \Gamma$. Since

$$
A_{n}^{\varepsilon}\left(U^{\prime}\right) U=\left[\begin{array}{c}
n \cdot u \\
p n-\left(H^{\prime} \cdot n\right) H+\left(\left(H^{\prime}+G\right) \cdot H\right) n \\
-\left(H^{\prime} \cdot n\right) u+(n \cdot u)\left(H^{\prime}+G\right)+\varepsilon H
\end{array}\right]
$$

by Lemma 3.1 (b ); $G \cdot n=0$ and the assumption $u^{\prime} \cdot n=0$, we get

$$
\left\{\begin{array}{l}
\text { (i ) } p n-\left(H^{\prime} \cdot n\right) H+\left(\left(H^{\prime}+G\right) \cdot H\right) n=0, \\
\text { (ii })-\left(H^{\prime} \cdot n\right) u+\varepsilon H=0 .
\end{array}\right.
$$

First taking inner product of (ii) with $n$ yields $H \cdot n=0$. Next taking inner product of (i ) with $n$ gives, by the assumption $H^{\prime} \times n=0$, we have $p=-G \cdot H$. The substitution of this into (i ) yields $\left(H^{\prime} \cdot n\right) H=0$. From the assumption $\inf _{x \in \Gamma}\left|H^{\prime} \cdot n\right| \geqq \tilde{c}$ on $[0, T]$, it follows that $H=0$. Hence using (i) and (ii) just proved, we can see $p=0$ and $u=0$. This completes the proof of (1).

Next we prove the statement of (2). If $U={ }^{t}(p, u, H) \in N$,

$$
\begin{aligned}
{ }^{t} U A_{n}^{\varepsilon}\left(U^{\prime}\right) U & =2\left[p(u \cdot n)+\left\{\left(H^{\prime}+G\right) \cdot H\right\}(u \cdot n)-\left(H^{\prime} \cdot n\right)(H \cdot u)\right] \\
& +\varepsilon|H|^{2}=\varepsilon|H|^{2} \geqq 0 .
\end{aligned}
$$

Namely $N$ is nonnegative with respect to $A_{n}^{\varepsilon}\left(U^{\prime}(t, x)\right)$ if $\varepsilon \geqq 0$. To show " maximally" part, we consider the linear subspaces properly incluuding $N$ which are divided into the following cases: $(\boldsymbol{\alpha})$ For the space generated by $N$ and the element such that $u \cdot n(x) \neq 0$ and $H \times n(x)=0$, we can choose $U=$ $(1,-n(x), n(x))$ in it, then ${ }^{t} U A_{n}^{\varepsilon}\left(U^{\prime}\right) U=-2<0$, because $G \cdot n=0$ from Lemma 3.1 (b). ( $\beta$ ) For the space generated by $N$ and the element such that $H \times n(x) \neq 0$ and $u \cdot n(x)=0$, we can choose $U=\left(1, \quad(\varepsilon+1) \tau / 2 \tilde{c}^{2}\right.$, $\tau /\left(H^{\prime} \cdot n(x)\right)$ in it, where $\tau$ is a unit vector orthogonal to $n(x)$, then ${ }^{t} U A_{n}^{\varepsilon}\left(U^{\prime}\right) U=-(\varepsilon+1) / \tilde{c}^{2}+\varepsilon /\left|H^{\prime} \cdot n(x)\right|^{2}<0$, because the assumption $\inf _{x \in \Gamma}\left|H^{\prime} \cdot n\right| \geqq \tilde{c} .(\gamma)$ For the space generated by $N$ and the elements such that $u \cdot n(x) \neq 0$ and $H \times n(x) \neq 0$, we can choose $U=(G \cdot \tau,-(\varepsilon+1) n(x) / G \cdot \tau, \tau)$ in it if $G \neq 0$, then ${ }^{t} U A_{n}\left(U^{\prime}\right) U=-3 \varepsilon-4<0$, because $H^{\prime} \times n=0$. If $G=0$, we can choose $U=(\varepsilon-1, n(x), \tau)$ in it, then ${ }^{t} U A_{n}\left(U^{\prime}\right) U=-\varepsilon-2<0$. Hence if $\varepsilon \geqq 0$, the null space of the boundary condition, $N$, is maximally nonnegative. The proof of Lemma 3.2 is completed. 
Thus the added terms $\varepsilon(\boldsymbol{n} \cdot \nabla) H_{\varepsilon}$ in the left side of $(3.9)_{\varepsilon}(\mathrm{c})^{\prime}$ make the boundary matrix nonsingular, keeping the boundary conditions maximally nonnegative.

\section{$\S 4$. Iteration Scheme.}

To define the iteration scheme for the initial boundary value problem (1.1)-(1.3), we first approximate the initial data $U_{0} \in H^{m}(\Omega)$ by the functions $U_{0}^{j} \in H^{m+2}(\Omega)$ that satisfy the compatibility conditions of order $m+1$. Before constructing these approximations of $U_{0}$, we take the molifier, $G^{k} \in$ $C^{\infty}([0, T] \times \bar{\Omega})$ of $G$ for $k=0,1, \cdots$, so that there exists a positive constant $\gamma^{\prime} \ll 1$ such that

$$
\sup _{t \in[0, T]}\left(\sum_{j=0}^{m}\left\|\partial_{t}^{j}\left(G^{k}-G\right)(t)\right\|_{m-j+1}+\left\|\partial_{t}^{m+1}\left(G^{k}-G\right)(t)\right\|_{1}\right)<\gamma^{\prime} 2^{-k}
$$

and $G^{k} \cdot n=0$ on $[0, T] \times \Gamma$ for $k=0,1, \cdots$. For this $G^{k}$, we construct the approximations of $U_{0},\left\{U_{0}\right\}_{j=0}^{\infty}$, as follows.

LEMMA 4.1. There exists a sequence $\left\{U_{0}^{j}\right\}_{j=0}^{\infty}$ in $H^{m+2}(\Omega)$ such that each $U_{0}^{j}$ satisfies the compatibility conditions of order $m+1$ in the following meanings :

$U_{0}^{0}$ satisfies the compatibility conditions of order $m+1$ for the equations (3.1) in which $G$ is replaced by $G^{0}$ and the boundary conditions (3.3). $U_{0}^{j}(j=1,2, \cdots)$ satisfies the compatibility conditions of order $m+1$ for the equations $A_{0}\left(U^{j-1}\right) \partial_{t} U+\sum_{i=1}^{3} A_{i}\left(U^{j-1}\right) \partial_{i} U+B U=F$ in which $G$ is replaced by $G^{j}$ and the boundary conditions (3.3), where $U^{j-1}$ satisfies that $\partial_{t}^{k} U^{j-1}(0)=\partial_{t}^{k} U_{0}^{j-1}$ for $k=1,2, \cdots, m+1$ and $\partial_{t}^{k} U_{0}^{j-1}$ denotes $k$-th time derivatives of $U_{0}^{j-1}$ which are determined in the preceding steps and the estimates hold:

$\left\|\mathrm{U}_{0}-\mathrm{U}_{0}^{j}\right\|_{m} \leqq \gamma 2^{-j}$ for $j=0,1, \cdots$, where $\gamma$ is a positive constant.

PROOF. In Lemma 3.3 of [9], such approximations has been constructed for the linear equations with the nonsingular boundary matrix. The method given there can be extended to the present case by the same arguments as in pp. 52-53 in [10], if we can check the relation

Range $M=$ Range $M\left(A_{n}\left(U_{0}\right)\right)^{k}$ on $\Gamma$ for $k=1,2, \cdots, m$, where $M$ is the matrix giving the boundary conditions (3.3):

$$
M=\left[\begin{array}{ccc}
0 & { }^{t} n & 0 \\
0 & 0 & n \times
\end{array}\right]
$$

where 
The Initial Boundary Value Problem for the Equations

$$
n \times=\left[\begin{array}{ccc}
0 & n_{3} & -n_{2} \\
-n_{3} & 0 & n_{1} \\
n_{2} & -n_{1} & 0
\end{array}\right] .
$$

Since $A_{n}\left(U_{0}\right)$ is a symmetric matrix, Range $\left(A_{n}\left(U_{0}\right)\right)^{k}=$ Range $A_{n}\left(U_{0}\right)$ for $k=1,2, \cdots$. So it is sufficient to show that

Range $M=$ Range $M A_{n}\left(U_{0}\right)$ on $\Gamma$.

We easily know that Range $M=R^{3}$. Since $G \cdot n=0$ on $\Gamma$, straightforward computations yield

$$
M A_{n}(U)=\left[\begin{array}{ccc}
|n| & 0 & h_{n} \\
0 & H_{n} & 0
\end{array}\right] \text { on } \Gamma
$$

where $h_{n}=\left(-(H \cdot n) n_{i}+|n|^{2}\left(H_{i}+G_{i}\right) \mid i \rightarrow 1,2,3\right)$ and $H_{n}=\left(n_{i}((H+G) \times n)_{j} \mid i\right.$ $\rightarrow 1,2,3, \mathrm{j} \downarrow 1,2,3)-(H \cdot n) n \times$. Therefore we can see from routine calculations Range $M A_{n}\left(U_{0}\right)=R^{3}$ on $\Gamma$, since $\inf _{x \in \Gamma}\left|H_{0} \bullet n\right| \geqq c_{2}$. Now the proof of Lemma 4.1 is completed.

Let us make some remarks which are necessary to construct an invariant subset for iteration scheme in $\S 5$. At first, let $R$ be an arbitrary small but fixed constant. Next, let $U={ }^{t}(p, u, H)$ be any function on $[0, T] \times \Omega$ satisfying

$$
\begin{aligned}
& \sup _{x \in \Omega}\left|p_{0}-p\right|<R, \sup _{x \in \Omega}\left|H_{0}-H\right|<R, \sup _{x \in \Omega}\left|u_{0}-u\right|<R, \\
& \text { for fixed } t \in[0, T] .
\end{aligned}
$$

Then, from the assumptions (1.4), (3.5) and (3.6) together with Lemma 3.1 (b), there exist a positive constant $k_{0}$, depending only on $c_{1}$, and an open covering, $\left\{\Omega_{i}\right\}_{i=0}^{N}$, of $\Omega$ with $\Omega_{0} \cap \Gamma=\phi, \Omega_{i} \cap \Gamma \neq \phi$ for $i=1,2, \cdots, N$, such that

$$
\begin{array}{ll}
\text { (a) } k_{0} I_{7} \leqq A_{0}(U(t)) \leqq k_{0}^{-1} I_{7} \text { in } \Omega, & \\
\text { (b) } \inf _{x \in \Omega \cap \Omega_{i}}|(H+G)(t) \cdot \boldsymbol{n}| \geqq c_{2} / 2 & \text { for } i=1,2, \cdots N \text {, } \\
\text { (c) } \sup _{x \in \Omega \cap \Omega_{i}}|u(t) \cdot \boldsymbol{n}| \leqq \tilde{c} & \text { for } i=1,2, \cdots N .
\end{array}
$$

Here $\boldsymbol{n}$ is the extended one and $c_{2}$ is the constant in (1.6), and $\tilde{c}$ a positive constant which will be determined later. We also may take $\gamma$ in Lemma 4.1 so that $\gamma=c^{-1} R / 2$ for $R$ in (4.1). Then the estimates for $\left\{U_{0}^{j}\right\}_{j=0}^{\infty}$ in Lemma 4. 1 become

$$
\left\|U_{0}-U_{0}^{j}\right\|_{m} \leqq c^{-1} R 2^{-j-1} \text { for } j=0,1, \cdots .
$$

Furthermore from the estimates of $G^{k}$ and $(4.2)(\mathrm{b})$, we can see that for 
any function $\mathrm{H}$ which satisfies (4.1) the following estimates hold:

$$
\inf _{x \in \Omega \cap \Omega_{i}}\left|\left(H+G^{k}\right)(t) \cdot \boldsymbol{n}\right| \geqq c_{2} / 4 \text { for } i=1,2, \cdots N, k=0,1, \cdots .
$$

Hereafter $\hat{H}^{j} \in H^{m+2}([0, T] \times \Omega)$ denotes the function in $[0, T] \times \Omega$ which is constructed by the same manner as (3.10), where we use $U_{0}^{j}$ instead of $U_{0}$. Then we rewrite the modified equations $(3.9)_{\varepsilon}$ in the matrix form, which corresponds to (3.7):

$$
A_{0}\left(U_{\varepsilon}\right) \partial_{t} U_{\varepsilon}+\sum_{i=0}^{3} A_{i, k}^{\varepsilon}\left(U_{\varepsilon}\right) \partial_{i} U_{\varepsilon}+B_{k}(t, x) U_{\varepsilon}=F_{\varepsilon, k}(t, x) .
$$

Here $G$ is replaced by $G^{k}$ and $U_{\varepsilon}={ }^{t}\left(p_{\varepsilon}, u_{\varepsilon}, H_{\varepsilon}\right)$. For the $A_{i, k}^{\varepsilon}\left(U_{\varepsilon}\right)$ and $B_{k}(t$, $x$ ), we define for convenience the following differential operator $L_{\varepsilon, k}(U)$ :

$$
L_{\varepsilon, k}(U)=A_{0}(U) \partial_{t}+\sum_{i=1}^{3} A_{i, k}^{\varepsilon}(U) \partial_{i}+B_{k}(t, x) .
$$

We also use the notation $F_{\varepsilon, k}^{j}(t, x)$ to denote the term $F_{\varepsilon, k}(t, x)$ with $\varepsilon(\boldsymbol{n}$. $\nabla) \hat{H}$ replaced by $\varepsilon(\boldsymbol{n} \cdot \nabla) \hat{H}^{j}$. We remark that this $F_{\varepsilon, k}^{j}(t, x)$ belongs to $H^{m+1}([0, T] \times \Omega)$.

Next we construct the first approximating solution $U^{0}$ of the iteration scheme as follows.

Lemma 4.2. There exists $U^{0}={ }^{t}\left(p^{0}, u^{0}, H^{0}\right) \in X_{m+1}(T)$ such that

$$
\left\{\begin{aligned}
u^{0} \cdot n=0, & H^{0} \times n=0 \text { on } \Gamma \times[0, T], \\
\partial_{t}^{k} U^{0}(0)= & \partial_{t}^{k} U_{0}^{0} \text { for } k=0,1, \ldots, m \text {, where } \partial_{t}^{k} U_{0}^{0} \text { denotes } k \text {-th time } \\
& \text { derivative at } t=0 \text { of a solution for the Cauchy problem } \\
& (3.1), \text { in which } G \text { is replaced by } G_{0}, \text { with the initial } \\
& \text { data } U_{0}^{0} .
\end{aligned}\right.
$$

REMARK 4. 1. It follows from (4.3) and (4.5) that there exists a positive constant $T^{\prime}$ so that for $R$ in (4.1)

$$
\sup _{x \in \Omega}\left|p_{0}-p^{0}\right|<R, \sup _{x \in \Omega}\left|H_{0}-H^{0}\right|<R, \sup _{x \in \Omega}\left|u_{0}-u^{0}\right|<R \text {, on }\left[0, T^{\prime}\right] .
$$

ProOf OF Lemma 4.2. Let $\varepsilon$ be a positive constant, and $U^{0}$ a solution of the following linear mixed problem:

$$
\left\{\begin{array}{l}
L_{\varepsilon, 0}\left(U_{0}^{0}\right) U^{0}=K_{\varepsilon}(t, x) \text { in }[0, T] \times \Omega, \\
\left.U^{0}\right|_{t=0}=U_{0}^{0} \text { in } \Omega, \\
u^{0} \cdot n=0, H^{0} \times n=0 \text { on }[0, T] \times \Gamma .
\end{array}\right.
$$

Here $K_{\varepsilon}$ is a function in $[0, T] \times \Omega$ which satisfies the following equality at $t=0$, 
The Initial Boundary Value Problem for the Equations

$$
\begin{aligned}
\partial_{t}^{k} K_{\varepsilon}(0) & =\partial_{t}^{k} F_{\varepsilon, 0}^{0}(0)-\left.\left[\partial_{t}^{k}, A_{0}\left(U^{0}\right)\right] \partial_{t} U^{0}\right|_{t=0} \\
& -\left.\sum_{j=1}^{3}\left[\partial_{t}^{k}, A_{j, 0}^{\varepsilon}\left(U^{0}\right)\right] \partial_{j} U^{0}\right|_{t=0} \text { for } k=0,1, \cdots, m,
\end{aligned}
$$

where [ , ] denotes commutator. The $K_{\varepsilon}$ is constructed by the same manner as (3.10); and belongs to $H^{m+1}([0, T] \times \Omega)$ since the right side of (4.5) belongs at least to $H^{m+1-k-1 / 2}(\Omega)$. Since $U^{0}(\mathrm{t})=U_{0}^{0}$, it follows, by inductions, from the definition of $F_{\varepsilon, 0}^{0}(0)$ and the above equality of $K_{\varepsilon}$ at $t=0$ that $\partial_{t}^{k} U^{0}(0)=\partial_{t}^{k} U_{0}^{0}$ for $k=1, \cdots, m$. Therefore, according to the results of the linear hyperbolic mixed problems, we can show from Lemma 3.2 and Lemma 4.1 together with $(4.2)$ (a), (4.3) and the inequality (2.1) that there exists a unique solution of the mixed problem (4.4) belonging to $X_{m+1}(T)$. (c. f. [9] and Appendix A of [10].) The proof of Lemma 4. 2 is completed. Now we can define iteration scheme.

$$
\left.\begin{array}{l}
\left\{\begin{array}{l}
\mathrm{L}_{\varepsilon_{j}, j}\left(\mathrm{U}_{\varepsilon_{j-1}}^{j-1}\right) \mathrm{U}_{\varepsilon_{j}}^{j}=\mathrm{F}_{\varepsilon_{j, j}}^{j} \quad \text { in }[0, T] \times \Omega, \\
\left.U_{\varepsilon_{j}}^{j}\right|_{t=0}=U_{0}^{j} \text { in } \Omega, \\
u_{\varepsilon_{i}}^{j} \cdot n=0, H_{\varepsilon_{j}}^{j} \times n=0
\end{array} \text { on }[0, T] \times \Gamma,\right.
\end{array}\right\}
$$

Here $U_{\varepsilon_{j}}^{j}={ }^{t}\left(p_{\varepsilon_{j}}^{j}, u_{\varepsilon_{j}}^{j}, H_{\varepsilon_{j}}^{j}\right), \quad U_{\varepsilon_{0}}^{0}=U^{0}$ in Lemma 4.2 and $\varepsilon_{j}=2^{-j} \theta(j)^{-1}$, where $\theta(j)$ is a positive monotone increasing function, tending to infinity as $j \rightarrow \infty$, such that $\left\|U_{0}^{j}\right\|_{m+2} \leqq \theta(j)\left\|U_{0}\right\|_{m}$.

REMARK 4.2. We can show from the construction of $\hat{H}^{j}$ and the definition of $\theta(j)$ that

$$
\varepsilon_{j}\left\|(\boldsymbol{n} \cdot \nabla) \hat{H}^{j}\right\|_{H^{m+1}([0, T] \times \Omega)} \leqq \varepsilon_{j} c\left\|U_{0}^{j}\right\|_{m+2} \leqq c 2^{-j}\left\|U_{0}\right\|_{m} .
$$

\section{$\S 5$. Proof of Theorem.}

To construct invariant set for iteration scheme, we define for positive constants $\tilde{T}$ and $M$

$S(\tilde{T}, M)=\left\{U={ }^{t}(p, u, H) \mid U \in X_{m+1}(\tilde{T}),\|U\|_{X_{m}(\tilde{T})} \leqq M, u \cdot n=0, H \times n=0\right.$ on $[0, \tilde{T}] \times \Gamma, U$ satisfies $(4.1)$ for all $t \in[0, \tilde{T}]\}$.

We now state the main Lemma.

Lemma 5.1. There exist positive constants $T_{*}$ and $M_{*}$ depending only on $\left\|U_{0}\right\|_{m}, k_{0}, c_{2},\|g\|_{Y_{m}(T)}, m$ and $\Omega$ such that if there exist a solution $U_{\varepsilon_{k}}^{k}$ of (4.6) ${ }_{k}$ for $k=0, \ldots, j-1$ which belongs to $S\left(T_{*}, M_{*}\right)$ then there exists a unique solution $U_{\varepsilon_{j}}^{j}$ of $(4.6)_{j}$ which again belongs to $S\left(T_{*}, M_{*}\right)$.

Proof. Suppose that $U_{\varepsilon_{j-1}}^{j-1} \in S_{(}\left(T^{\prime}, M\right)$, where $T^{\prime}$ is the positive constant in REMARK 4.1 and $M$ is a positive constant such that $\left\|U^{0}\right\|_{X_{m}\left(T^{\prime}\right)} \leqq M$. 
Then, it is easy to see from Lemma 3.2, Lemma 4. 1, Lemma 4.2 and (4.2) (a) for $U_{\varepsilon_{j-1}}^{j-1}$ on $\left[0, T^{\prime}\right]$ that there exists a unique local in time solution $U_{\varepsilon_{j}}^{j}$ for the linear equations (4.6) $)_{j}$ belonging to $X_{m+1}\left(T^{\prime}\right)$.

Therefore we have only to show the following key a priori estimate for $U_{\varepsilon_{j}}^{j}$ :

$$
\left\|U_{\varepsilon_{j}}^{j}(t)\right\|_{m} \leqq\left(k_{a}+k_{b} t\right) \exp \left(k_{c} t\right) \text { for } 0 \leqq t \leqq \min \left(T^{\prime}, T_{a}, 1\right),
$$

where $k_{a}, k_{b}$ and $k_{c}$ are positive constants independent of $j$. The constant $k_{a}$ depends only on $\left\|U_{0}\right\|_{m}, k_{0}, c_{2},\|g\|_{Y_{m}(T)}, m$ and $\Omega$. Moreover, $k_{b}$ and $k_{c}$ depend only on $M,\left\|U_{0}\right\|_{m},\|g\|_{Y_{m}(T)}, k_{0}, c_{2}, m$ and $\Omega$. Furthermore, $T_{a}$ is a positive constant depending only on $M,\left\|U_{0}\right\|_{m},\|g\|_{Y_{m}(T)}, k_{0}, c_{2}, \varepsilon_{0}, m$ and $\Omega$.

Then, taking $M_{*}=\max \left(6 k_{a}, M\right)$ and $T_{1}=\min \left(k_{b}^{-1}\left(M_{*}\right) k_{a}, k_{c}^{-1}\left(M_{*}\right)\right.$, $\left.T_{a}\left(M_{*}\right), T^{\prime}, 1\right)$, where $k_{b}\left(M_{*}\right), k_{c}\left(M_{*}\right), T_{a}\left(M_{*}\right)$ denotes $k_{b}, k_{c}$ and $T_{a}$ with $M$ replaced by $M_{*}$, we can see from the estimate (5.1) that

$$
\left\|U_{\varepsilon_{j}}^{j}\right\|_{X_{m}\left(T_{1}\right)} \leqq M_{*} .
$$

Therefore this estimate together with (2.1) leads that there exists a positive constant $T_{*}$ independent of $j$ such that

$$
\begin{aligned}
& \sup _{x \in \Omega}\left|p_{0}^{j}-p_{\varepsilon_{j}}^{j}\right|<R / 2, \sup _{x \in \Omega}\left|H_{0}^{j}-H_{\varepsilon_{j}}^{j}\right|<R / 2, \\
& \sup _{x \in \Omega}\left|u_{0}^{j}-u_{\varepsilon_{j}}^{j}\right|<R / 2 \text { on }\left[0, T_{*}\right],
\end{aligned}
$$

because for example $p_{\varepsilon_{j}}^{j}-p_{0}^{j}=\int_{0}^{t} \partial_{\tau} p_{\varepsilon_{j}}^{j}(\tau) \mathrm{d} \tau$ and $\sup _{t \in\left[0, T_{*}\right]}\left\|\partial_{t} p_{\varepsilon_{j}}^{j}(t)\right\|_{m-1} \leqq M_{*}$. Accordingly, from (4.3) and (2.1) we see that

$$
\begin{aligned}
& \sup _{x \in \Omega}\left|p_{0}-p_{\varepsilon_{j}}^{j}\right|<R, \sup _{x \in \Omega}\left|H_{0}-H_{\varepsilon_{j}}^{j}\right|<R, \\
& \sup _{x \in \Omega}\left|u_{0}-u_{\varepsilon_{j}}^{j}\right|<R \text { on }\left[0, T_{*}\right]
\end{aligned}
$$

and $U_{\varepsilon_{j}}^{j} \in S\left(T_{*}, M_{*}\right)$.

Now we begin to establish the estimate (5.1). For simplicity we use such abbreviations as $U^{j}$ for $U_{\varepsilon_{j}}^{j}$ and use $k_{i}\left(M, k_{0}, \cdots\right)(i=1,2, \cdots)$ as a positive constant independent of $j$ which depends on $M, k_{0}, \cdots$.

At first, we give the estimate of $\operatorname{div} H^{j}$ which is uniform with respect to $j$ by making use of a special structure of $(3.9)_{\varepsilon}$.

Note that the equations of $H^{j}$ corresponding to $(3.9)_{\varepsilon}(\mathrm{c})^{\prime}$ are

$$
\begin{aligned}
& \left(\partial_{t}+\left(u^{j-1} \cdot \nabla\right)\right) H^{j}-\left(\left(H^{j-1}+G^{j}\right) \cdot \nabla\right) u^{j}+\left(H^{j-1}+G^{j}\right) \operatorname{div} u^{j} \\
& +\left(u^{j-1} \cdot \nabla\right) G^{j}+\varepsilon_{j}(\boldsymbol{n} \cdot \nabla) H^{j}=\varepsilon_{j}(\boldsymbol{n} \cdot \nabla) \hat{H}^{j}-\partial_{t} G^{j} .
\end{aligned}
$$

Accordingly, it follows from a well-known formula from vector analysis that 
The Initial Boundary Value Problem for the Equations

of Ideal Magneto-Hydrodynamics

$$
\begin{aligned}
& \left(\partial_{t}+\left(u^{j-1} \cdot \nabla\right)\right) H^{j}-\left(u^{j} \cdot \nabla\right)\left(H^{j-1}+G^{j}\right)+u^{j} \operatorname{div}\left(H^{j-1}+G^{j}\right) \\
& -\operatorname{curl}\left(u^{j} \times\left(H^{j-1}+G^{j}\right)\right)+\left(u^{j-1} \cdot \nabla\right) G^{j}+\varepsilon_{j}(\boldsymbol{n} \cdot \nabla) H^{j} \\
& =\varepsilon_{j}(\boldsymbol{n} \cdot \nabla) \hat{H}^{j}-\partial_{t} G^{j} .
\end{aligned}
$$

Therefore taking the divergence of these equations and noting that div curl= 0 , we get

(5.3) $\partial_{t} \operatorname{div} H^{j}+\left(u^{j-1} \cdot \nabla\right) \operatorname{div} H^{j}+\varepsilon_{j}(\boldsymbol{n} \cdot \nabla) \operatorname{div} H^{j}+l$. o. $t$.

$=\operatorname{div}\left(\varepsilon_{j}(\boldsymbol{n} \cdot \nabla) \hat{H}^{j}-\partial_{t} G^{j}\right)$,

where $l$. o. $t$. means the lower order terms of $H^{j}$ and $u^{j}$. We remark that their coefficients contain at most first order derivatives of $U^{j-1}$ and second order derivatives of $G^{j}$ because the terms which contain second order derivatives of $H^{j-1}$ are canceled each other. Operating $\partial^{\alpha}$ on (5.3) (this $\partial^{\alpha}$ contains both time and space derivatives), multiplying (5.3) by $2 \partial^{\alpha} \mathrm{div} H^{j}$, summing over all $\alpha$ wth $|\alpha| \leqq m-1$, and applying integration by parts and inequalities (2.1), (2.2), we obtain by Lemma 3.1 (c)

$\partial_{t}\left\|\operatorname{div} H^{j}(t)\right\|_{m-1}^{2}$

$\leqq\left(k_{1}\left(M,\|g\|_{Y_{m}(T)}, m, \Omega\right)\left\|U^{j}(t)\right\|_{m}\right.$

$\left.+\left(k_{2}\left(\|g\|_{Y_{m}(T)}, \quad m, \quad \Omega\right)+\varepsilon_{j}\left\|\operatorname{div}\left((\boldsymbol{n} \cdot \nabla) \hat{H}^{j}(t)\right)\right\|_{m-1}\right)\right)\left\|\operatorname{div} H^{j}(t)\right\|_{m-1}$

$-\varepsilon_{j} \sum_{|\alpha| \leq m-1} \int_{\Gamma}\left|\partial^{\alpha} \operatorname{div} H^{j}\right|^{2} \mathrm{~d} \Gamma$,

since $u^{j-1} \cdot n=0$ on $\Gamma$. Therefore we get the following estimate of $\operatorname{div} H^{j}$ which is crucial in this proof:

$$
\begin{aligned}
& \left\|\operatorname{div} H^{j}(t)\right\|_{m-1} \leqq k_{3}\left(\left\|U_{0}\right\|_{m}, m, \Omega\right)+k_{2} t \\
& +\int_{0}^{t}\left(k_{1}\left\|U^{j}(\tau)\right\|_{m}+\varepsilon_{j} \| \operatorname{div}\left((\boldsymbol{n} \cdot \nabla) \hat{H}^{j}(\tau) \|_{m-1}\right) \mathrm{d} \tau \quad \text { for } 0 \leqq t \leqq T^{\prime} .\right.
\end{aligned}
$$

Because of (4.7), this estimate is uniform with respect to $j$.

Next, let us take a partition of unity, $\left\{\phi_{i}\right\}_{i=0}^{N}$, with respect to the open covering $\left\{\Omega_{i}\right\}_{t=0}^{N}$ in (4.2) (b) such that $\sum_{i=0}^{N} \phi_{i}^{2}(x)=1$ in a neighborhood of $\Omega$ and each $\phi_{i}$ is $C^{\infty}$-function with compact support in $\Omega_{i}$. Then we may suppose that $\Gamma \cap \Omega_{i}(i \neq 0)$ is given by the smooth function $\psi_{i}$ such that

$$
\begin{aligned}
& \Gamma \cap \Omega_{i}=\left\{\left(x_{1}, x_{2}, x_{3},\right) \mid x_{3}=\psi_{i}\left(x_{1}, x_{2}\right)\right\}, \\
& \Omega \cap \Omega_{i}=\left\{\left(x_{1}, x_{2}, x_{3}\right) \mid x_{3}=\psi_{i}\left(x_{1}, x_{2}\right)\right\} .
\end{aligned}
$$

For this $\psi_{i}$, we define the change of independent variables in $\Omega_{i}$ :

$$
\left\{\begin{array}{l}
y_{1}=x_{1}, \\
y_{2}=x_{2}, \\
y_{3}=x_{3}-\psi_{i}\left(x_{1}, x_{2}\right) .
\end{array}\right.
$$


Then we see easily that there exists a positive smooth function $\varkappa_{i}(\mathrm{x})$ in $\Omega_{i}$ $(i=1,2, \cdots, N)$ such that

$$
\varkappa_{i}(x) \boldsymbol{n}(x)=\left(-\partial_{1} \psi_{i},-\partial_{2} \psi_{i}, 1\right) \text { on } \Omega \cap \Omega_{i},
$$

and $\Gamma \cap \Omega_{i}$ is mapped to the subset of $\left\{\left(y_{1}, y_{2}, y_{3}\right) \mid y_{3}=0\right\}$. Moreover we change the dependent variables $\phi_{i} U^{j}$ by an orthogonal matrix valued transform $T_{i}(x)$ which makes the boundary operator $M$ constant on $\Gamma$ (c.f. Proposition 3.1 of [6]]), and rewrite for simplicity this new variables $T_{i} \phi_{i} U^{j}$ by $\tilde{U}^{j}$ in $\Omega_{i}$. Then note that this $\tilde{U}^{j}$ satisfies the following equations in $\Omega_{i}$ :

$$
\begin{aligned}
& \tilde{A}_{0}\left(U^{j-1}\right) \partial_{t} \tilde{U}^{j}+\tilde{A}_{1, j}^{\varepsilon_{j}}\left(U^{j-1}\right) \partial_{y_{1}} \tilde{U}^{j}+\tilde{A}_{2, j}^{\varepsilon_{j}}\left(U^{j-1}\right) \partial_{y_{2}} \tilde{U}^{j} \\
& +\varkappa_{i} \tilde{A}_{n, j}^{\varepsilon_{j}}\left(U^{j-1}\right) \partial_{y_{3}} \tilde{U}^{j}+\tilde{B}_{j} \tilde{U}^{j}-\sum_{k=1}^{2} \tilde{A}_{k, j}^{\varepsilon_{j}}\left(U^{j-1}\right) \partial_{y_{k}}\left\{\phi_{i} T_{i}\right\} U^{j} \\
& -\varkappa_{i} \tilde{A}_{n, j}^{\varepsilon_{j}}\left(U^{j-1}\right) \partial_{y_{3}}\left\{\phi_{i} T_{i}\right\} U^{j}=\tilde{F}_{\varepsilon_{j}, j}^{j},
\end{aligned}
$$

where $\partial_{y_{i}}=\partial / \partial y_{i}, \tilde{A}_{k, j}^{\varepsilon_{j}}\left(U^{j-1}\right)=T_{i} \tilde{A}_{k, j}^{\varepsilon_{j}}\left(U^{j-1}\right)^{t} T_{i}$ for $k=0,1,2, \boldsymbol{n}, \tilde{B}_{j}=T_{i} B_{j}{ }_{j} T_{i}$, $\tilde{F}_{\varepsilon j, j}^{j}=T_{i} \phi_{i} F_{\varepsilon_{j}, j}^{j}$. Applying tangential derivative operator $\partial_{\text {tan }}^{\alpha}$ with $|\alpha| \leqq m$ to the equations (5.7), we get

$$
\begin{aligned}
& \tilde{A}_{0}\left(U^{j-1}\right) \partial_{t} \tilde{U}_{\tan }^{j, \alpha}+\tilde{A}_{1, j}^{\varepsilon_{j}}\left(U^{j-1}\right) \partial_{y_{1}} \tilde{U}_{\tan }^{j, \alpha}+\tilde{A}_{2, j}^{\varepsilon_{j}}\left(U^{j-1}\right) \partial_{y_{2}} \tilde{U}_{\tan }^{j, \alpha} \\
& +\varkappa_{i} \tilde{A}_{n, j}^{\varepsilon_{j}}\left(U^{j-1}\right) \partial_{y_{3}} \tilde{U}_{\tan }^{j, \alpha}+\tilde{B}_{j} \tilde{U}_{\tan }^{j, \alpha}+l . \quad o . \quad t .=\partial_{\tan }^{\alpha} \tilde{F}_{\varepsilon_{j}, j}^{j},
\end{aligned}
$$

where $\tilde{U}_{\tan }^{j, \alpha}=\partial_{\tan }^{\alpha} \tilde{U}^{j}, \partial_{\tan }^{\alpha}=\partial_{t}^{\alpha_{0}} \partial_{y_{1}}^{\alpha_{1}} \partial_{y_{2}}^{\alpha_{2}}$ for $\alpha=\left(\alpha_{0}, \alpha_{1}, \alpha_{2}\right),|\alpha|=\alpha_{0}+\alpha_{1}+\alpha_{2}$. And here $l$. o. $t$. means the lower order terms of $U^{j}$ with their coefficients which contain at most $m$-th order derivatives of $U^{j-1}$ and $(m+1)$-th order derivatives of $G^{j}$. Multiplying (5.8) by $\tilde{U}_{\tan }^{j, \alpha}$ and integrating over $x$ lead to

$$
\begin{aligned}
& \partial_{t}\left(\tilde{U}_{\tan }^{j, \alpha}, \tilde{A}_{0}\left(U^{j-1}\right) \tilde{U}_{\tan }^{j, \alpha}\right)_{0} \\
& =-\int_{y_{3}=0} \tilde{U}_{\tan }^{j, \alpha} \varkappa_{i} \tilde{A}_{n_{,}}^{\varepsilon_{j}}\left(U^{j-1}\right) \tilde{U}_{\tan }^{j, \alpha} \mathrm{d} \Gamma+\left(\tilde{U}_{\tan }^{j, \alpha},\left\{\partial_{t} \tilde{A}_{0}\left(U^{j-1}\right)\right.\right. \\
& +\partial_{y_{1}} \tilde{A}_{1, j}^{\varepsilon_{j}}\left(U^{j-1}\right)+\partial_{y_{2}} \tilde{A}_{2,{ }_{j}}^{\varepsilon_{j}}\left(U^{j-1}\right)+\partial_{y_{3}}\left(\varkappa_{i} \tilde{A}_{n, j}^{\varepsilon_{j} j_{j}}\left(U^{j-1}\right)\right)
\end{aligned}
$$

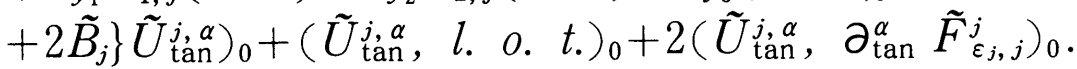

Here we note that $\tilde{U}_{\tan }^{j, \alpha} \varkappa_{i} \tilde{A}_{n, j}^{\varepsilon_{j}^{j}}\left(U^{j-1}\right) \tilde{U}_{\tan }^{j, \alpha} \geqq 0$ on $y_{3}=0$, because the null space of the boundary conditions is nonnegative with respect to $A_{n, j}^{\varepsilon_{j}}\left(U^{j-1}\right)$ (c.f. Lemma 3.2). We also note that $M \partial_{\tan }^{\alpha} \tilde{U}^{j}=\partial_{\tan }^{\alpha} M \tilde{U}^{j}=0$ on $y_{3}=0$ since the transformed $M$ is constant. Summing over all $\alpha$ with $|\alpha| \leqq m$, applying the inequalities (2.1) and (2.2), we can get by Lemma 3.1 (c)

$$
\begin{aligned}
& \partial_{t} \sum_{|\alpha| \leqq m}\left(\tilde{A}_{0}\left(U^{j-1}\right) \tilde{U}_{\text {tan }}^{j, \alpha}, \quad \tilde{U}_{\text {tan }}^{j, \alpha}\right)_{0} \\
& \leqq\left(k_{4}\left(\|g\|_{Y m(T)}, m, \Omega\right)+\varepsilon_{j}\left\|T_{i} \boldsymbol{\phi}_{i}(\boldsymbol{n} \cdot \nabla) \hat{H}^{j}(t)\right\|_{m, \tan }\right. \\
& \left.+k_{5}\left(M,\|g\|_{Y_{m}(T)}, \quad m, \Omega\right)\left\|U^{j}(t)\right\|_{m}\right)\left\|\tilde{U}^{j}(t)\right\|_{m, \tan },
\end{aligned}
$$


where $\left\|\tilde{U}^{j}(t)\right\|_{m, \tan }^{2}=\sum_{|\alpha| \leq m}\left\|\partial_{\tan }^{\alpha} \tilde{U}^{j}(t)\right\|_{0}^{2}$. Therefore (4.2)(a) yields

$$
\begin{aligned}
& \left\|\tilde{U}^{j}(t)\right\|_{m, \tan } \\
& \leqq k_{6}\left(\left\|U_{0}\right\|_{m}, m, \Omega\right)+k_{0}^{-1} k_{4} t \\
& +k_{0}^{-1} \int_{0}^{t}\left(k_{5}\left\|U^{j}(\tau)\right\|_{m}+\varepsilon_{j}\left\|T_{i} \phi_{i}(\boldsymbol{n} \cdot \nabla) \hat{H}^{j}(\tau)\right\|_{m}\right) \mathrm{d} \tau \quad \text { for } 0 \leqq t \leqq T^{\prime} .
\end{aligned}
$$

Here and hereafter we use $\tilde{U}^{j}$ again to represent $\phi_{i} U^{j}$ instead of $T_{i} \phi_{i} U^{j}$.

Next we shall give the estimates of normal derivatives of $\tilde{U}^{j}$ which are independent of $j$. For this purpose we should note that the rank of the boundary matrix $\tilde{A}_{n, j}^{\varepsilon_{j}}\left(U^{j-1}\right)$ is 6 on $\Gamma$ because of (4.4) for $\cdot H_{\varepsilon_{j-1}}^{j-1}$. Therefore we can see the estimate for the normal derivatives of $\tilde{U}^{j}$ by returning to the equations (5.7) and combining the uniform estimates (5.5) and (5.10). To see this procedure precisely, we first note that divergent operator can be locally written as

$$
\begin{aligned}
\operatorname{div} \tilde{H}^{j}= & \partial_{y_{1}} \tilde{H}_{1}^{j}+\partial_{y_{2}} \tilde{H}_{2}^{j}+\partial_{y_{3}} \tilde{H}_{3}^{j}-\partial_{1} \psi_{i} \partial_{y_{3}} \tilde{H}_{1}^{j} \\
& -\partial_{2} \psi_{i} \partial_{y_{3}} \tilde{H}_{2}^{j} \quad \text { in } \Omega \cap \Omega_{i} .
\end{aligned}
$$

Hence we get the identity :

$$
\begin{aligned}
& \boldsymbol{\varkappa}_{i}\left(\boldsymbol{n}_{1} \partial_{y_{3}} \tilde{H}_{1}^{j}+\boldsymbol{n}_{2} \partial_{y_{3}} \tilde{H}_{2}^{j}+\boldsymbol{n}_{3} \partial_{y_{3}} \tilde{H}_{3}^{j}\right) \\
& =\operatorname{div} \tilde{H}^{j}-\partial_{y_{1}} \tilde{H}_{1}^{j}-\partial_{y_{2}} \tilde{H}_{2}^{j} \quad \text { in } \Omega \cap \Omega_{i} .
\end{aligned}
$$

Moreover, the equations for $\tilde{U}^{j}$ are written as

$$
\begin{aligned}
& \varkappa_{i} \tilde{A}_{n, j}^{\varepsilon_{j}}\left(U^{j-1}\right) \partial_{y_{3}} \tilde{U}^{j}=-\left\{A_{0}\left(U^{j-1}\right) \partial_{t} \tilde{U}^{j}+\sum_{k=1}^{2} \tilde{A}_{k, j}^{\varepsilon_{j}^{j}}\left(U^{j-1}\right) \partial_{y_{k}} \tilde{U}^{j}\right\} \\
& +\sum_{k=1}^{2} \tilde{A}_{k, j}^{\varepsilon_{j}}\left(U^{j-1}\right) \partial_{y_{k}}\left\{\phi_{i}\right\} U^{j}+\varkappa_{i} \tilde{A}_{n_{, j}}^{\varepsilon_{j}}\left(U^{j-1}\right) \partial_{y_{3}}\left\{\phi_{i}\right\} U^{j}+\tilde{F}_{\varepsilon_{j}, j}^{j} \text { in } \Omega \cap \Omega_{i} .
\end{aligned}
$$

Then the equations of the second, the third, the fourth lines of (5.12) and the identity (5.11) can be written as

$$
\begin{aligned}
& \Xi_{\alpha} \partial_{y_{3}}{ }^{t}\left(\tilde{p}^{j}, \tilde{H}_{1}^{j}, \tilde{H}_{2}^{j}, \tilde{H}_{3}^{j}\right) \\
& =(4 \text {-dimensional vector valued functions consisting of the right } \\
& \text { side of }(5.11) \text { and corresponding lines of }(5.1) \text { and } \rho\left(p^{j-1}\right)\left(u^{j-1}\right. \text {. } \\
& \text { n) } \left.\partial_{y_{3}} u_{i}^{j} \text { for } i=1,2,3\right) .
\end{aligned}
$$

Here

$$
\boldsymbol{\Xi}_{\alpha}=\varkappa_{i}\left(\begin{array}{cc}
\boldsymbol{n} & D_{\boldsymbol{n}}^{j-1} \\
0 & { }^{\boldsymbol{n}} \boldsymbol{n}
\end{array}\right],
$$

where, $D_{n}^{j-1}=\left(-\left(H^{j-1}+G^{j}\right) \cdot \boldsymbol{n} \delta_{l, k}+\boldsymbol{n}_{k}\left(H_{l}^{j-1}+G_{l}^{j}\right) \mid l \rightarrow 1,2,3, k \downarrow 1,2,3\right)$. Then from (4.4) and the assumption $\sup _{x \in \Omega}\left|H^{j-1}-H_{0}\right|<R$, it follows that 


$$
\begin{gathered}
\inf _{x \in \Omega \cap \Omega_{i}}\left|\left(H^{j-1}+G^{j}\right) \cdot \boldsymbol{n}\right| \geqq c_{2} / 4 \text { on }\left[0, T^{\prime}\right], \text { which implies } \\
\quad \operatorname{det} \boldsymbol{\Xi}_{\alpha}=\mathcal{\varkappa}_{i}^{4}\left(\left(H^{j-1}+G^{j}\right) \cdot \boldsymbol{n}\right)^{2} \geqq \mathcal{\varkappa}_{i}^{4} c_{2}^{2} / 16 \\
\quad \text { in }\left[0, T^{\prime}\right] \times \Omega \cap \Omega_{i} .
\end{gathered}
$$

Next note that the equations of the first, the fifth, the sixth line of $(5.12)$ can be written as

$$
\begin{aligned}
& \Xi_{\beta} \partial_{y_{3}}{ }^{t}\left(\tilde{u}_{1}^{j}, \tilde{u}_{2}^{j}, \tilde{u}_{3}^{j}\right) \\
& =(3 \text {-dimensional vector valued functions consisting of the right } \\
& \text { side of corresponding lines of }(5.12), \\
& \varepsilon_{j} \partial_{y_{3}} \tilde{H}_{i}^{j}+\left(u^{j-1} \cdot \boldsymbol{n}\right) \partial_{y_{3}} \tilde{H}_{i}^{j} \text { for } i=1,2 \text { and } \\
& \left.\rho^{-1}\left(p^{j-1}\right) \rho_{p}\left(p^{j-1}\right)\left(u^{j-1} \cdot \boldsymbol{n}\right) \partial_{y 3} p^{j}\right) .
\end{aligned}
$$

Here

$$
\Xi_{\beta}=\boldsymbol{\varkappa}_{i}\left(\begin{array}{ccc}
\boldsymbol{n}_{1} & \boldsymbol{n}_{2} & \boldsymbol{n}_{3} \\
-\left(H^{j-1}+G\right) \cdot \boldsymbol{n}+\left(H_{1}^{j-1}+G_{1}\right) \boldsymbol{n}_{1} & \left(H_{1}^{j-1}+G_{1}\right) \boldsymbol{n}_{2} & \left(H_{1}^{j-1}+G_{1}\right) \boldsymbol{n}_{3} \\
\left(H_{2}^{j-1}+G_{2}\right) \boldsymbol{n}_{1} & -\left(H^{j-1}+G\right) \cdot \boldsymbol{n}+\left(H_{2}^{j-1}+G_{2}\right) \boldsymbol{n}_{2} & \left(H_{2}^{j-1}+G_{2}\right) \boldsymbol{n}_{3}
\end{array}\right),
$$

where $G=G^{j}$. Then $\inf _{x \in \Omega \cap \Omega_{i}}\left|\left(H^{j-1}+G^{j}\right) \cdot \boldsymbol{n}\right| \geqq c_{2} / 4$ on $\left[0, T^{\prime}\right]$ again yields

$\operatorname{det} \boldsymbol{\Xi}_{\beta}=\varkappa_{i}^{2}\left(\left(H^{j-1}+G^{j}\right) \cdot \boldsymbol{n}\right)^{2} \geqq \mathcal{\varkappa}_{i}^{2} c_{2}^{2} / 16$ in $\left[0, T^{\prime}\right] \times \Omega \cap \Omega_{i}$.

Then, taking $\tilde{c}$ in (4.2)(c) small enough for given $\left\|U_{0}\right\|_{m},\|g\|_{Y_{m}(T)}, c_{2}^{-1}$, and $\varepsilon_{0}$, taking a positive constant $T_{a}$ small enough for this $\tilde{c}$ and $M$, and then applying the mean value theorem and the inequalities (2.1) and (2.2), we can show from (5.13) and (5.14) that

$$
\begin{aligned}
& \left\|\tilde{U}^{j}(t)\right\|_{m} \\
& \leqq k_{7}\left(c_{2}^{-1},\left\|U^{j-1}(t)\right\|_{m-1},\|g\|_{Y(T)}, \quad m, \Omega\right) \\
& \times\left(\left\|\operatorname{div} H^{j}(t)\right\|_{m-1}+\left\|\tilde{U}^{j}(t)\right\|_{m, \tan }+\left\|U^{j}(t)\right\|_{m-1}\right) \\
& \leqq k_{7} \times\left(\left\|\operatorname{div} H^{j}(t)\right\|_{m-1}+\left\|\tilde{U}^{j}(t)\right\|_{m, \tan }\right. \\
& \left.+k_{8}\left(\left\|U_{0}\right\|_{m}, m, \Omega\right)+\int_{0}^{t}\left\|U^{j}(\tau)\right\|_{m} \mathrm{~d} \tau\right) \quad \text { for } 0 \leqq t \leqq \min \left(T^{\prime}, T_{a}\right) .
\end{aligned}
$$

Here the constant $k_{7}$ depends smoothly on $\left\|U^{j-1}(t)\right\|_{m-1}$. Therefore we get

$$
\begin{aligned}
& k_{7}\left(c_{2}^{-1},\left\|U^{j-1}(t)\right\|_{m-1},\|g\|_{Y_{m}(T)}, m, \Omega\right) \\
& \leqq k_{9}\left(c_{2}^{-1},\left\|U_{0}\right\|_{m},\|g\|_{Y_{m}(T)}, m, \Omega\right)+k_{10}\left(c_{2}^{-1}, M,\|g\|_{Y_{m}(T)}, m, \Omega\right) t
\end{aligned}
$$

On the other hand, in the same arguments as in the case of Cauchy problem, we can show from (4.7) 
(5.16) $\quad\left\|\phi_{0} U^{j}(t)\right\|_{m} \leqq k_{11}+k_{12} t+k_{13} \int_{0}^{t}\left\|U^{j}(\tau)\right\| \mathrm{d} \tau$

for $0 \leqq t \leqq \min \left(T^{\prime}, 1\right)$,

where $k_{11}=k_{11}\left(\left\|U_{0}\right\|_{m}, m, \Omega\right), k_{12}=k_{12}\left(M, k_{0}^{-1},\left\|U_{0}\right\|_{m},\|g\|_{Y m(T)}, m, \Omega\right)$ and $k_{13}$ $=k_{13}\left(M, k_{0}^{-1},\|g\|_{Y m(T)}, m, \Omega\right)$.

Accordingly, since

$$
\left\|U^{j}(t)\right\|_{m}^{2} \leqq\left\|\phi_{0} U^{j}(t)\right\|_{m}^{2}+\sum_{i=1}^{N}\left\|T_{i} \phi_{i} U^{j}(t)\right\|_{m}^{2},
$$

it follows from (4.7), (5.5), (5.10), (5.15), and Gronwall's inequality that

$$
\left\|U^{j}(t)\right\|_{m} \leqq\left(k_{14}+k_{15} t\right) \exp \left(k_{16} t\right) \text { for } 0 \leqq t \leqq \min \left(T^{\prime}, T_{a}, 1\right),
$$

where $k_{14}=k_{14}\left(c_{2}^{-1},\left\|U_{0}\right\|_{m},\|g\|_{Y m(T)}, m, \Omega\right), k_{15}=k_{15}\left(M, k_{0}^{-1}, c c_{2}^{-1},\left\|U_{0}\right\|_{m}\right.$, $\left.\|g\|_{Y m(T)}, m, \Omega\right)$ and $k_{16}=k_{16}\left(M, k_{0}^{-1}, c_{2}^{-1},\|g\|_{Y m(T)}, m, \Omega\right)$. Therefore the proof of Lemma 5.1 is completed.

Next we show that the sequence $\left\{U_{\varepsilon_{j}}^{j}\right\}_{j=0}^{\infty}$ converges strongly in $X_{0}(T)$ for a sufficiently small constant $T$.

LEMMA 5.2. There exists a positive constant $T_{0}$ such that

$$
\sup _{t \in\left[0, T_{0}\right]} \sum_{j=1}^{\infty}\left\|U_{\varepsilon_{j}}^{j}(t)-U_{\varepsilon_{j-1}}^{j-1}(t)\right\|_{0}<\infty .
$$

Proof. For simplicity, we also use $U^{j}$ for $\dot{U}_{\varepsilon_{j}}^{j}$ in this proof. Direct computations yields the equations for $U^{j}-U^{j-1}$ :

$$
\begin{aligned}
& A_{0}\left(U^{j-1}\right) \partial_{t}\left(U^{j}-U^{j-1}\right)+\sum_{i=1}^{3} A_{i, j}^{\varepsilon_{j}^{j}}\left(U^{j-1}\right) \partial_{i}\left(U^{j}-U^{j-1}\right) \\
& +B_{j}\left(U^{j}-U^{j-1}\right)-\left(F_{\varepsilon_{j}, j}^{j}-F_{\varepsilon_{j-1}, j-1}^{j-1}\right)=-A_{0}\left(U^{j-1}\right) \sum_{i=1}^{3}\left(\tilde{A}_{i, j}^{\varepsilon_{j}}\left(U^{j-1}\right)-\right. \\
& \left.\tilde{A}_{1, j_{-1}}^{\varepsilon_{-1} 1}\left(U^{j-2}\right)\right) \partial_{i} U^{j-1}-A_{0}\left(U^{j-1}\right)\left(A_{0}^{-1}\left(U^{j-1}\right) B_{j}-A_{0}^{-1}\left(U^{j-2}\right) B_{j-1}\right) U^{j-1} \\
& +A_{0}\left(U^{j-1}\right) F_{\varepsilon_{j-1}, j-1}^{j-1}\left(A_{0}^{-1}\left(U^{j-1}\right)-A_{0}^{-1}\left(U^{j-2}\right)\right),
\end{aligned}
$$

where $\tilde{A}_{i, k}^{\varepsilon_{k}}\left(U^{k-1}\right)=A_{0}^{-1}\left(U^{k-1}\right) A_{i, k}^{\varepsilon_{k}}\left(U^{k-1}\right)$. Noting that the estimates (4.7) and

$$
\left\|U^{j-1}\right\|_{X_{m}\left(T_{*}\right)} \leqq M,\left\|U^{j-2}\right\|_{X_{m}\left(T_{*}\right)} \leqq M, \quad k_{0} I_{7} \leqq A_{0}\left(U^{j-1}\right) \leqq k_{0}^{-1} I_{7}
$$

in $\left[0, T_{*}\right] \times \Omega$, we conclude by standard energy estimates for symmetric hyperbolic systems and the mean value theorem that

$$
\begin{aligned}
& \sup _{\tau \in\left[0, T_{0}\right]}\left\|U^{j}(\tau)-U^{j-1}(\tau)\right\|_{0} \leqq\left(\left\|U_{0}^{j}-U_{0}^{j-1}\right\|_{0}\right. \\
& \left.+c^{\prime}\left(2^{-j}+\sup _{\tau \in\left[0, T_{0}\right]}\left\|U^{j-1}(\tau)-U^{j-2}(\tau)\right\|_{0}\right) T_{0}\right) \exp c^{\prime \prime} T_{0}
\end{aligned}
$$


for $T_{0} \leq T_{*}$, where $c^{\prime}$ and $c^{\prime \prime}$ are positive constants depending only on $M$, $\left\|U_{0}\right\|_{m},\|g\|_{Y_{m}(T)}, k_{0}, m$, and $\Omega$. Therefore taking $T_{0}$ small enough, we have, by (4.3)

$$
\sup _{\tau \in\left[0, T_{0}\right]}\left\|U^{j}(\tau)-U^{j-1}(\tau)\right\|_{0} \leqq \alpha \sup _{\tau \in\left[0, T_{0}\right]}\left\|U^{j-1}(\tau)-U^{j-2}(\tau)\right\|_{0}+\beta_{j},
$$

where $0<\alpha<1$ and $\left\{\beta_{j}\right\}_{j=2}^{\infty}$ with $\sum_{j=2}^{\infty}\left|\beta_{j}\right|<\infty$. This estimate immediately yields (5.18).

By Lemma 5.1 and Lemma 5.2, together with the sobolev space interpolation inequalities (c.f. pp. 39 of [8]), we can conclude that there exists a function $U$ such that for any positive constant $\delta$ the sequence $\{U$ $\left.\left.{ }_{\varepsilon}^{j}\right\}_{j}\right\}_{j=0}^{\infty}$ converges to $U$ strongly in $H^{m-\delta}(\Omega)$ uniformly on $\left[0, T_{0}\right]$ and the sequence $\left\{\partial_{t} U_{\varepsilon_{j}}^{j}\right\}_{j=0}^{\infty}$ converges to $\partial_{t} U$ strongly in $H^{m-1-\delta}(\Omega)$ unifomly on $[0$, $T_{0}$ ]. Accordingly, we get

$$
U \in C^{0}\left(0, T_{0} ; H^{m-\delta}(\Omega)\right) \cap C^{1}\left(0, T_{0} ; H^{m-1-\delta}(\Omega)\right) .
$$

Because of $m \geqq 3$, this $U$ is a classical solution of the initial boundary value problem (1.1)-(1.3).

The uniqueness of the solution of (1.1)-(1.3) follows directly from the next a priori estimate.

Lemma 5.3. Assume that $U$ and $U^{\prime}={ }^{t}\left(p^{\prime}, u^{\prime}, H^{\prime}\right)$ are solutions of (1.1) belonging to $X_{m}(T)(m \geqq 3)$ which satisfy only the solid boundary condition $u \cdot n=0$ on $[0, T] \times \Gamma$.

Then the following a priori estimate holds:

$$
\begin{aligned}
& \left\|U(t)-U^{\prime}(t)\right\|_{0}^{2} \leqq c_{3} \exp \left(c_{4} t\right)\left\{\left\|U(0)-U^{\prime}(0)\right\|_{0}^{2}\right. \\
& \left.+\int_{0}^{t} \int_{\Gamma}\left|(H(\tau) \cdot n)\left\{\left(H(\tau)-H^{\prime}(\tau)\right) \cdot\left(u(\tau)-u^{\prime}(\tau)\right)\right\}\right| \mathrm{d} \Gamma \mathrm{d} \tau\right\}
\end{aligned}
$$

for $0 \leqq t \leqq T$, where $c_{3}, c_{4}$ are positive constants which depend on $|\rho|_{L^{\infty}}$ and $|\nabla U|_{L^{\infty}},\left|\nabla U^{\prime}\right|_{L^{\infty}}$, respectively. Here $|\cdot|_{L^{\infty}}$ denotes essential supremum norm on $[0, T] \times \Omega$.

PRoof. Direct computations yield the equations for $U-U^{\prime}$ :

$$
\begin{aligned}
A_{0}(U) \partial_{t}\left(U-U^{\prime}\right) & +\sum_{j=1}^{3} A_{j}(U) \partial_{j}\left(U-U^{\prime}\right)=\left(A_{0}(U)\right) \partial_{t} U^{\prime} \\
& +\sum_{j=1}^{3}\left(A_{j}\left(U^{\prime}\right)-A_{j}(U)\right) \partial_{j} U^{\prime}
\end{aligned}
$$

where $A_{0}(U), A_{j}(U)$ are the matrices of (3.7) with $G=0$.

Note that under the condition $u \cdot n=0$ on $\Gamma$ 
The Initial Boundary Value Problem for the Equations

$$
\int_{\Gamma}^{t}\left(U-U^{\prime}\right) A_{n}(U)\left(U-U^{\prime}\right) \mathrm{d} \Gamma=2 \int_{\Gamma} H \cdot n\left\{\left(H-H^{\prime}\right) \cdot\left(u-u^{\prime}\right)\right\} \mathrm{d} \Gamma
$$

(c. f. proof of Lemma 3.2). Then (5.20) follows from the standard method for symmetric hyperbolic systems.

Finally we shall remove $\delta$ in (5.19). Since $\left\{U_{\varepsilon_{j}}^{j}\right\}_{j=0}^{\infty}$ converges to $U$ strongly in $H^{m-\delta}(\Omega)$ uniformly on $\left[0, T_{0}\right]$ and $\left\|U_{\varepsilon_{j}}^{j}\right\|_{X_{m}\left(T_{0}\right)} \leqq M$ for $j=0,1, \cdots$ and since $\left(H^{m-\delta}(\Omega)\right)^{\prime}$ is dense in $\left(H^{m}(\Omega)\right)^{\prime}$, (the dual space of $H^{m}(\Omega)$; c.f. pp. 84 of [7]), we can show by an elementary argument that

$$
U \in C_{w}\left(0, T_{0} ; H^{m}(\Omega)\right),
$$

where $C_{w}$ means continuity on the interval $\left[0, T_{0}\right]$ with values in the weak topology of $H^{m}(\Omega)$ (c. f. pp. 40 of [8]).

Next, we obtain from (1.1)(c), (1.5) and div curl=0 that

$$
\operatorname{div} H(t)=0 \quad \text { for } 0 \leqq t \leqq T_{0} .
$$

Furthermore, applying the tangential molifier for the principal parts of the equations corresponding to (5.7), carrying out the similar estimates to (5.9) for $\phi_{i} T_{i} U(i=1, \cdots N)$, we obtain the following estimate: for some positive constant $k$,

$$
\begin{aligned}
& \left\|\phi_{i} T_{i} U(t)\right\|_{m, \tan }^{2}-\left\|\phi_{i} T_{i} U(s)\right\|_{m, \tan }^{2} \\
& \leqq k\left(M,\|g\|_{Y_{m}(T)}, k_{0}^{-1}, m, \Omega\right)|t-s| \text { for } 0 \leqq t, s \leqq T_{0}, \quad i=1, \cdots, N,
\end{aligned}
$$

since $\|U\|_{X_{m}\left(T_{0}\right)} \leqq M$. Accordingly, we get from (5.21)

$$
\partial_{\tan }^{\alpha}\left(\phi_{i} T_{i} U\right) \in C\left(0, T_{0} ; L^{2}(\Omega)\right) \text { with }|\alpha| \leqq m \text { for } i=1, \cdots, N .
$$

Hence, by using the similar relations to (5.13) and (5.14) for $U$ we can show that

$$
\phi_{i} U \in C\left(0, T_{0} ; H^{m}(\Omega)\right) \text { for } i=1, \cdots, N,
$$

since $T_{i}^{-1}$ are smooth. Since $\phi_{i}$ are smooth and since $\phi_{0} U \in C\left(0, T_{0}\right.$; $H^{m}(\Omega)$ ), we get

$$
U \in C\left(0, T_{0} ; H^{m}(\Omega)\right)
$$

Therefore, by returning to the equations (1.1) we get

$$
U \in X_{m}\left(T_{0}\right) \text {. }
$$

Now the proof of THEOREM 1.1 is completed.

Acknowledgements: I express my many thanks to Professor Rentaro Agemi, who guided me this problem. I also would like to express my sincere thanks to Professor Taira Shirota for his continuous encouragement and 
valuable suggestions.

\section{References}

[1] R. AGEMI, The initial boundary value problem for inviscid barotropic fluid motion, Hokkaido Math. J. 10 (1981), 156-182.

[2] L. CATtABRiga, Su un problema al cotorno relativo al sistema di equazioni di stokes, Rend. Sem. Mat. Univ Padova 31 (1961), 308-340.

[ 3 ] D. G. EBIN, The initial boundary value problem for subsonic fluid motion, Comm. Pure Appl. Math. 32 (1979), 1-19.

[ 4 ] A. Friedman, Partial Differential Equations, Krieger-Huntington, New York, 1976.

[ 5 ] L. HöRMANDER, Linear Partial Differential Operators, Springer-Verlag, Berlin, Heidelberg, New York, 1976.

[ 6 ] M. IKAwA, Mixed problem for a hyperbolic system of the first order, Publ. R. I. M. S., Kyoto Univ. 7 (1971/72), 427-454.

[ 7 ] J. L. Lions, E. MAGENES, Problèms aux limites non homogènes et applications, vol. 1, Dunod, Paris, 1968.

[ 8 ] A. MAJDA, Compressible flow and systems of conservation laws in several space variables, Applied math. sciences 53, Springer-Verlag, New York, 1984.

[ 9 ] J. RAUCH and F. MASSEY III, Differentiability of solutions to hyperbolic initial-boundary value problems, Trans. A. M. S. 189 (1974), 303-318.

[10] S. SCHOCHET, The compressible Euler equations in a bounded domain: Existence of solutions and incompressible limit, Comm. Math. Phys. 104 (1986), 49-75.

[11] H. BEIRÃo DA VeIGA. On the barotropic motion of compressible perfect fluids, Ann. Sc. Normale Sup. Pisa 8 (1981), 317-351.

Department of Mathematics, Nara Women's University 